\title{
S-wave superconductivity near a surface
}

\author{
K. Tanaka ${ }^{1,2}$ and F. Marsiglio ${ }^{3}$ \\ ${ }^{1}$ Department of Physics and Engineering Physics, University of Saskatchewan, \\ Saskatoon, SK, Canada S7N 5E2 \\ ${ }^{2}$ Materials Science Division, Argonne National Laboratory, \\ 9700 South Cass Avenue, Argonne, IL 60439 U.S.A. \\ ${ }^{3}$ Department of Physics, University of Alberta, Edmonton, Alberta, Canada T6G 2J1
} (published in Physica C 384 (2003) 356)

\begin{abstract}
We study the superconducting order parameter near a surface with the Bogoliubov-de Gennes formalism. For definiteness we use the attractive Hubbard model. Near a surface, the order parameter and the density distribution exhibit "Friedellike" oscillations. Although the local density of states is quite different from that in the bulk, the energy gap in the spectrum on a surface is almost the same as the bulk value. In the low-density limit, however, the energy gap tends to vanish on a surface.
\end{abstract}

\section{INTRODUCTION}

Experimental probes of various properties of superconductors can generally be classified into two categories bulk and surface probes. Tunneling and photoelectron spectroscopy (PES) are examples in the second category, and while PES has only recently been utilized heavily to investigate superconductivity [1], tunneling has long been an important tool for determining the energy gap [2] and electron-phonon related structure [3] in superconductors. Through comparison with results from bulk probes, it has been well established that the superconducting order parameter in conventional superconductors remains robust at surfaces.

The purpose of this paper is to investigate the extent to which this statement is theoretically understood. In order to do so, in the context of conventional s-wave superconductivity, we adopt a minimal model to describe s-wave superconductivity, namely the attractive Hubbard model. This choice allows us to explore various aspects of the problem that otherwise might be difficult to study: electron density dependence and weak-to-strong coupling tendencies, to name a few. The tight-binding and lattice features of the this model also make it amenable to numerical treatment. The presence of surfaces can be included in this model simply by requiring that electrons cannot hop beyond the surfaces, e.g., by imposing open boundary conditions (OBC) as opposed to periodic boundary conditions $(\mathrm{PBC})$.

The paper is organized as follows. In the next section we outline the formalism used in this study. We simply solve the Bogoliubov-de Gennes (BdG) equations [4], as described in a previous publication [5]. We also compare results with those obtained from the Anderson prescription $[6,5]$, for which a brief review is provided. In the following section we present results for the order parameter as a function of distance from a surface for various parameter regimes. It is clear that a large spatial variation occurs, dependent on coupling strength and electron density. We also examine the local density of states as a function of distance from the surface, and find significant variation close to the surface. In this work we focus our study on one dimension, in which the impact of a surface can be visualized clearly. The effects of dimensionality on the "Friedel-like" oscillations are discussed, and also some results for the local density of states are shown for a two-dimensional system.

\section{FORMULATION}

The use of the Bogoliubov-de Gennes (BdG) equations $[4,8]$ (or a corresponding quasi-classical formulation [9]) is required to solve for the superconducting order parameter in cases where the order parameter varies with position. This will generally occur in the vicinity of a defect or impurity, but first and foremost, will always occur near a surface. Even if the surface does not modify the material parameters, its existence causes an abrupt change in the effective pair potential, and hence the order parameter will vary in its vicinity.

What governs the length scale of the variation in the order parameter as one moves away from the surface? Three length scales enter the problem (we leave out the actual thickness of the sample, which enters in the Tomasch-Rowell experiment [10], or in nanograin superconductors). These are (i) the lattice spacing, $a$, (ii) the coherence length, $\xi_{0}$, and (iii) a measure of the inter-electron spacing, say, $k_{F}^{-1}$, where $k_{F}$ is the Fermi wavevector. Near half-filling (i) and (iii) coincide; in the low-density limit, we will find that (iii) governs the order parameter variation. In all cases, one can observe a 'healing length', over which the order parameter attains its bulk value. This is qualitatively described by the coherence length, determined here with the BCS expression:

$$
\xi=\sqrt{\left\langle R^{2}\right\rangle}
$$


where $\left\langle R^{2}\right\rangle$ is the mean square radius of an electron pair $[11,12]$. Strictly speaking, as defined this length has little to do with coherence (defined for a single pair). However, at low temperatures, to which we confine this study, this tends to coincide with the Ginzburg-Landau coherence length, which more properly describes the length scale of fluctuations in the order parameter away from its mean value.

The BdG equations are $[8,5]$ :

$$
\begin{aligned}
& E_{l} u_{l}(i)=\sum_{i^{\prime}} A_{i i^{\prime}} u_{l}\left(i^{\prime}\right)+V_{i} u_{l}(i)+\Delta_{i} v_{l}(i) \\
& E_{l} v_{l}(i)=-\sum_{i^{\prime}} A_{i i^{\prime}} v_{l}\left(i^{\prime}\right)-V_{i} v_{l}(i)+\Delta_{i}^{*} u_{l}(i)
\end{aligned}
$$

where

$$
A_{i i^{\prime}}=-t \sum_{\delta}\left(\delta_{i^{\prime}, i-\delta}+\delta_{i^{\prime}, i+\delta}\right)-\delta_{i i^{\prime}} \mu .
$$

The self-consistent potentials, $V_{i}$ and $\Delta_{i}$, are given by

$$
\begin{aligned}
\Delta_{i} & =|U| \sum_{l} u_{l}(i) v_{l}^{*}(i)\left(1-2 f_{l}\right) \\
V_{i} & =-|U| \sum_{l}\left[\left|u_{l}(i)\right|^{2} f_{l}+\left|v_{l}(i)\right|^{2}\left(1-f_{l}\right)\right] .
\end{aligned}
$$

The index $l$ is used to label the eigenvalues (there are $2 N$ of them), the index $i$ to label the sites (1 through $N)$, and the composite eigenvector is given by $\left(\begin{array}{l}u_{l} \\ v_{l}\end{array}\right)$, of total length $2 N$. The sums in Eqs. $(5,6)$ are over positive eigenvalues only. The $f_{l}$ is the Fermi function, with argument $\beta E_{l}$, where $\beta=1 / k_{B} T$ with $T$ the temperature, and $k_{B}$ the Boltzmann constant. The single-site electron density, $n_{i}$, is given through Eq. (6) by $V_{i}=-|U| n_{i} / 2$. As usual, the parameters $t$ and $|U|$ are the hopping integral and attractive coupling strength, $\mu$ is the chemical potential, and the subscript ' $\delta$ ' in Eq. (4) refers to nearest neighbour sites in units of the lattice spacing.

These equations are solved by iteration until full selfconsistency is achieved. If (as is often the case) a given electron density is desired, a second iteration loop is inserted to vary the chemical potential $\mu$, until the required average density $n \equiv \frac{1}{N} \sum_{i} n_{i}$ is attained. The quasiparticle amplitudes are used to calculate the local density of states (LDOS) given by

$A_{i}(\omega)=\sum_{l}\left[\left|u_{l}(i)\right|^{2} \delta\left(\omega-E_{l}\right)+\left|v_{l}(i)\right|^{2} \delta\left(\omega+E_{l}\right)\right]$.

We will make reference to solutions to the Anderson equations in what follows. As described in the original paper [6] and explained for the attractive Hubbard model in Ref. [5], these are a BCS-like set of equations for an inhomogeneous system, for which solutions are required for the non-interacting problem. For tight-binding systems with open boundary conditions, these solutions can be found analytically. Once the single-particle states are obtained, the BCS-like equations can be solved much more easily than their BdG counterparts. As shown previously [5] and further in this work, they provide an excellent qualitative description of the position dependence of the order parameter and the density of states, for a fraction of the numerical cost for BdG.

\section{RESULTS}

\section{A. BdG vs. Anderson}

In the absence of a surface or impurity, momentum is a good quantum number, and the BCS gap equations [13] can be solved. For the attractive Hubbard model, the resulting order parameter turns out to be constant in momentum space as well as in real space. The presence of a surface breaks translational invariance, so that both the order parameter and the density distribution become dependent on position close to the surface. In particular they exhibit "Friedel-like" oscillations. In Figs. 1 and 2 , this is demonstrated for the order parameter for a 64-site system with $\mathrm{OBC}$, where site 1 and 64 represent the surfaces. As mentioned above, the only distinction in our tight-binding formalism is that there is no hopping matrix element connecting the surface sites to outside of the chain. Further refinements, such as altering the hopping matrix elements or the attractive interaction for sites near the surface, are possible; however we do not explore these possibilities here. Unlike the Ginzburg-Landau approaches, we do not impose boundary conditions on the order parameter itself: the surface behaviour of the order parameter is determined by the BdG equations themselves.

In Figs. 1 and 2, the BdG and Anderson results are shown with solid and dashed curves, respectively. Figure 1 shows the order parameter $\Delta_{i}$ (scaled by the siteaveraged value) as a function of site number $i$ for half filling, for various coupling strengths. At half filling, the order parameter is peaked at the surface and oscillates as one moves in towards the bulk. These oscillations decay over the scale roughly determined by the coherence length $\xi$, beyond which the order parameter relaxes to its bulk value. For sufficiently large samples, the bulk value is the same as that achieved for the homogeneous case obtained with PBC. This can be seen clearly in Fig. 1, with $\xi$ becoming longer as the coupling strength is decreased. An exception is the case with $|U|=1.1 t$, where we encounter finite size effects - the system is not quite large enough to allow relaxation of the order parameter to its bulk value.

The "Friedel-like" oscillations in the order parameter are a reflection of the single-particle wave function at the 
Fermi level. For a given density $n$, the oscillation period in site number is given by $\pi / k_{F} a$, where $a$ is the lattice spacing, and $k_{F}$ and $n$ are connected by the usual Pauli consideration. To illustrate this, we plot the order parameter with $|U|=1.1 t$ for various values of density $n$ in Fig. 2. As $n$ decreases, $k_{F}$ decreases and the period becomes longer, as is indicated by the bar in each panel. Note moreover that the coherence length $\xi$, for the given coupling strength $|U|=1.1 t$, decreases monotonically from $n=1.0$ to 0.25 : this is shown in Fig. 3(a), where we plot $\xi$ as a function of electron density $n$. In Fig. 3(b) $\xi$ for half filling is plotted as a function of coupling strength $|U|$. In these figures, we have also included some analytical forms valid in the weak-coupling limit $\left(\xi_{B C S}\right)$, and in (b), one in the strong-coupling limit as well. Note, particularly in (b) the excellent agreement of the analytical forms with $\xi$ in the two extremes. It is clear that in both Figures 1 and 2 the Anderson prescription (dashed curves) captures the essential features of the "Friedel-like" oscillations in the order parameter. This is also the case for the electron density distribution (not shown).

In Fig. 4 we examine the local density of states calculated with both the BdG and Anderson formalisms. The delta functions in Eq. (7) have each been replaced by a normalized Gaussian, and the smoothing width for the results shown in all the LDOS figures is $0.1 t$. It can be seen that the Anderson prescription reproduces the BdG results remarkably well in all details. In this figure the LDOS at various sites is shown for the same system as in Fig. 1 but with $|U| / t=2.0$. Near a surface the LDOS is quite different from that in the bulk (as represented by the LDOS in the middle of the sample, or by the average DOS over all sites). Although the overall LDOS as a function of energy is very different from the bulk DOS, the energy gap in the spectrum on the surface is almost the same as that in the bulk (compare the BdG results for site 1 and 32). On the other hand, it is intriguing that the energy gap is larger at every second site (site 2 and 4 in Fig. 4): this statement applies only to half filling. For quarter filling, for example, the energy gap is slightly larger at every fourth site. The Anderson results reproduce these features correctly, and their agreement with the BdG results becomes better further away from the surface. Note, however, the absence of coherence peaks in the more accurate $\mathrm{BdG}$ results at the surface position.

\section{B. Friedel-like oscillations}

Next we study the "Friedel-like" oscillations in more detail. In Fig. 5 the density distribution $n_{i}$ as a function of site number $i$ is shown for a 64-site chain (OBC, $|U| / t=1.5)$ for various values of the average electron density $n$. In this figure the density distribution obtained from the BdG results (solid curves) is compared with the density distribution for the noninteracting case (dotted curves). Except for half filling, the density distributions in both the superconducting and normal states exhibit oscillations, with period determined by $k_{F}^{-1}$. In the superconducting state the oscillations are smoothed as one moves away from the surface, as was the case with the order parameter (see Fig. 2). This distance over which the electron density becomes smooth is the same as for the order parameter, and is loosely given by the coherence length $\xi$. The exceptional case of half filling $(n=1.0)$ has an electron density that is independent of site position, due to the particle-hole symmetry present at half filling (see next subsection, however).

As seen in Fig. 5, the density distribution (for nonzero $U)$ sometimes has higher amplitude near a surface, particularly close to half filling. This behaviour is not to be confused with the decay of the "Friedel-like" oscillations in the order parameter. The former simply follows the non-interacting distribution, which results from the particle-in-a-box problem subject to the Pauli exclusion principle. With OBC the single-particle states are standing waves, and the sum of their probability distribution tends to be higher near a surface near half filling.

While the electron-electron interaction is local in this model, in two and three dimensional systems, the "Friedel-like" oscillations appear in somewhat different ways from one dimension (1D). The difference stems mainly from two factors. One is that unlike in $1 \mathrm{D}$, with OBC, most of the single-particle energy levels are degenerate in higher dimensions. As a result, interference occurs among degenerate single-particle states at the Fermi level, and the order parameter and density distribution can exhibit quite complicated structure. In two dimensions (2D) at half filling, however, it is relatively simple due to the structure of the Fermi surface. We illustrate this in Fig. 6, where the order parameter is plotted for a $20 \times 20$-site system for $|U| / t=2.5$ and 1.5. At half filling, the order parameter shows the "Friedel-like" oscillations, where it is peaked along the diagonal sites and largest near the corners. As a function of decreasing $|U|$ (increasing $\xi$ ), the oscillations extend towards the middle of the sample. These oscillations, as seen in Fig. 6, can be explained by the interference of single-particle wave functions. In the zero-coupling limit $(|U| \rightarrow 0)$, Eq. (5) reduces to $\Delta_{i} \simeq|U|(1 / 2) \sum_{\mathbf{k}_{F}} u_{\mathbf{k}_{F}}(i) v_{\mathbf{k}_{F}}^{*}(i)$ at half filling at zero temperature, and $u_{\mathbf{k}_{F}}(i) \simeq v_{\mathbf{k}_{F}}(i) \simeq u_{\mathbf{k}_{F}}^{0}(i)$. Here $u_{\mathbf{k}_{F}}^{0}(i)$ is the single-particle wave function (analytical solution [5]) for the Fermi momentum $\mathbf{k}_{F}=\left(k_{x}, k_{y}\right)$, and the $\simeq$ denotes that the oscillatory behaviour is similar. In fact the amplitude of the hole-like part decreases with $|U|$ for electron-like states and vice-versa for the electronlike part.. Using the relation $k_{y}=-k_{x}+\pi$ on the Fermi surface to eliminate $k_{y}$, and approximating the sum by an integral over $k_{x}$, we can calculate $\Delta_{i}$ analytically. Thus 


$$
\Delta_{i} \simeq \Delta\left[1+\frac{1}{2}\left(\delta_{y_{i}, x_{i}}+\delta_{y_{i},-x_{i}}\right)\right],
$$

where $x_{i}, y_{i}$ are the $x$ and $y$ coordinates of site $i$, and $\Delta$ is a small number. Clearly $\Delta_{i}$ is larger along the diagonals $\left(x_{i}= \pm y_{i}\right)$. We have obtained the BdG solutions for $N=32^{2}$ at half filling as a function of $|U|$, and found that for very weak coupling (e.g., $|U| / t=0.1$ ), the above equation indeed describes the order parameter structure.

For three dimensional systems, the largest size we have examined is $N=12^{3}$. For coupling strengths small enough to see the "Friedel-like" oscillations, however, this size is still small - there are only 12 sites on one side and the surfaces occupy a large portion of the system - so that the order parameter is dominated by finite size effects. We have calculated the three-dimensional version of Eq. (8) numerically, and found that it qualitatively explains the oscillatory structure of the order parameter in the weak-coupling limit.

As $n$ becomes smaller than 1, the interference pattern changes. As seen above, in $2 \mathrm{D}$, for $n \simeq 1$, the Fermi surface is approximately $k_{y}=-k_{x}+\pi$ and the oscillations in $\Delta_{i}$ as well as $n_{i}$ are along several sites around the diagonal. Away from half filling, the oscillations occur everywhere along the surfaces. Figure 7 shows the order parameter and density distribution for $N=20^{2}$ and $|U| / t=2$ at quarter filling. In the low-density limit, both $n_{i}$ and $\Delta_{i}$ are suppressed on and near the surfaces.

Another factor that distinguishes one dimension from higher dimensions is the dependency of nesting on dimensionality. In 1D, the Fermi surface is perfectly nested for all values of $n$. In 2D and 3D this is the case only at half filling, and this means that away from half filling, "Friedel-like" oscillations can be suppressed compared with 1D. We have not identified any noticeable difference in this sense between $1 \mathrm{D}$ and $2 \mathrm{D}$. For $3 \mathrm{D}$, we have examined this aspect by placing a nonmagnetic impurity which shifts the chemical potential at that site and using PBC. In this way a disturbance that breaks translational invariance is one site, as opposed to all the surfaces. In Fig. 8 we show the order parameter for $N=11^{3}, n=0.9$, and $|U| / t=2$, in the first layer (top) and the second layer (bottom). The impurity potential is $0.5 t$ in the middle of the first layer. The basic features caused by an impurity are the same as in 1D [5]: in the first layer $\Delta_{i}$ is suppressed at the impurity site and shows the "Friedel-like" oscillations around it. The size-dependent electron density, $n_{i}$ (not shown), behaves similarly, except that for an attractive potential, it is peaked at the impurity site. The $\Delta_{i}$ is peaked right below the impurity site in the second layer and suppressed in the third layer, and so on. However, the extent to which the oscillations propagate is smaller compared with $1 \mathrm{D}$ and $2 \mathrm{D}$. This is the case within the layer where the impurity is, as seen in Fig. 8. Moreover, the oscillation amplitudes decrease quickly as one moves away from that layer: note that in Fig. 8 the $z$-axis range for the second layer is half of that for the first layer.

In the strong-coupling limit, at half filling in any dimension, the order parameter is larger on the surfaces (largest at the corners) and more or less flat inside. In Fig. 9 we show $\Delta_{i}$ for $N=12^{3}$ and $|U| / t=2$ at half filling, for the first four layers. While $\Delta_{i}$ is larger on the surfaces (the first layer and peripherals of each layer), some oscillations near the surfaces can be seen.

\section{CDW}

For the half-filled attractive Hubbard model with nearest neighbour hopping on a translationally invariant system, the superconducting (SC) and charge-density-wave (CDW) states are degenerate. The presence of an impurity tends to make a CDW state more favourable, and one might expect that a surface has the same effect. This is not necessarily the case, however. With an even number of sites $N$, with $\mathrm{OBC}$, the $\mathrm{SC}$ and $\mathrm{CDW}$ states are degenerate at half filling. Moreover, the state with constant density distribution discussed above is not the only $\mathrm{SC}$ solution. When the density distribution of a CDW state is used as input, the BdG equations converge to a SC state with a similar density distribution, and this state has the same energy as the one with constant density. The order parameter structure is the same for both states, but the overall magnitude is smaller for the former. For any average density, the BdG equations always converge to a SC solution.

The situation is different, however, for an odd- $N$ system with surfaces. The BdG equations converge to a $\mathrm{SC}$ solution at any density that corresponds to an odd number of electrons, e.g., at half filling. For an even number of electrons, the BdG equations either do not converge at all, or converge to a CDW solution. This presumably is caused by the incommensurability of the electron number (all paired) and the number of sites. The same CDW solution for an even number of electrons is obtained by solving the Hartree equations (no superconducting order parameter). With $N+1$ electrons the system is equivalent to an $N+1$ system with PBC at half filling, with a strong repulsive impurity at one site where the electron density becomes zero. While the Hartree equations usually do not converge for an odd electron number, we were able to find a CDW solution at half filling with odd $N$. It is interesting to note that unlike even $N$, the CDW state has a lower energy than the SC state, and we did not find a SC state with an oscillating density distribution.

In Fig. 10(a) we show the average density $n$ vs. chemical potential ("dressed" with the Hartree energy), obtained by BdG for $1 \mathrm{D}$ with $N=15$ with $|U| / t=1$. The plateaus correspond to CDW states, where the superconducting order parameter is zero. For stronger coupling and larger system size, the plateaus tend to disappear, 
but the BdG equations pick up the $\mathrm{CDW}$ solutions at certain densities. In Fig. 10(b) the density distribution is shown for a CDW state in $1 \mathrm{D}$ with $N=63,|U| / t=0.5$, and $n=18 / 63$. In both (a) and (b), the BdG and Anderson results are plotted with solid and dashed curves, respectively. Although the Anderson results are similar to the $\mathrm{BdG}$ results, they are not $\mathrm{CDW}$ solutions: the density distribution is the same as the one in the noninteracting case.

\section{Local density of states}

To distinguish the features that are due to the opening of a superconducting gap from those that are due to the single-particle states, we compare, in Fig. 11, the LDOS (the same BdG results as shown in Fig. 4) with that in the noninteracting case, for various sites near the surface. Interestingly, the noninteracting LDOS has a gap-like feature at every second site (e.g., site 2 and 4 ) near a surface (again this is the case only for half filling). This is consistent with a larger gap at these sites in the superconducting state.

As seen in Figs. 4 and 11, the energy gap as it appears in the LDOS on the surface is not very different from the one in the bulk, despite the fact that both the order parameter and the density distribution behave quite differently on and near the surface. However, the BCS coherence factors, which give rise to peaks in the density of states just beyond the gap (as seen in the LDOS for site 32 in Fig. 11), are barely existent near the surface; for half filling, particularly at the even-numbered sites. For an orbital structure much more complicated than the single-band one-dimensional model adopted here, these effects would have to be considered when interpreting STM or ARPES data.

The site variation of the LDOS also depends on electron density. In Fig. 12 we plot the LDOS at the surface of a 64-site chain for various average electron densities. We have solved the BdG equations self-consistently for $|U| / t=2.0$ and for the given values of electron density. The resulting LDOS is compared to that calculated in the bulk (we simply averaged over all sites, which gives a result identical to that obtained in the interior, or that obtained with PBC). Particularly in the low-density limit large differences occur: the bulk DOS always has a welldefined energy gap, with remnants of the BCS coherence peaks, whereas the LDOS on the surface does not. This occurs mainly because in the low-density limit, the electron density at the surface sites is significantly lower than in the bulk. This is most apparent in Fig. 5, where the electron density (even in the non-interacting limit) is considerably lower near the surface than in the bulk. This is true even when we include repulsive Coulomb interactions between electrons, as we have verified by direct calculation in the low-density limit. In other words,
Coulomb interactions do not affect the inhomogeneous electron distribution as much as one might think because we are in the dilute limit.

In $2 \mathrm{D}$ and $3 \mathrm{D}$, the LDOS behaves in a similar way as in $1 \mathrm{D}$, as one moves away from a corner along the diagonal. In Fig. 13 the LDOS for $N=32^{2}$ and $|U| / t=2.5$ at quarter filling is shown for several sites near the surfaces.

\section{SUMMARY}

We have calculated the spatially inhomogeneous order parameter and local density of states (LDOS) for a very simple model exhibiting s-wave superconductivity, as a function of coupling strength and electron density. We have used the Bogoliubov-de Gennes formalism and solved the resulting equations self-consistently, including both the anomalous and normal effective potentials. We find that both the order parameter and the density distribution vary significantly as a function of position near the surface. The details of this variation are dependent on both the electron density and the coupling strength. The LDOS shows a similar variation. If one can somehow preferentially tunnel electrons into the surface or subsurface layers, this variation may be observable [14]. Here again, details will be dependent on the specifics of the model, i.e., the character of the orbitals involved in the superconductivity as well as the symmetry of the superconducting order parameter. We intend to further investigate these issues in future work.

\section{ACKNOWLEDGMENTS}

We thank Matthias Eschrig and Stuart Trugman for stimulating discussions. This research was supported by the Natural Sciences and Engineering Research Council of Canada and the Canadian Institute for Advanced Research.

[1] See, for example, D.W. Lynch and C.G. Olsen, Photoemission Studies of High-Temperature Superconductors, (Cambridge University Press, Cambridge, 1999).

[2] I. Giaever, Phys. Rev. Lett. 5464 (1960).

[3] J.M. Rowell, P.W. Anderson, and D.E. Thomas, Phys. Rev. Lett. 10334 (1963).

[4] P. G. de Gennes, Superconductivity of Metals and Alloys (W.A. Benjamin, Inc. New York, 1966).

[5] K. Tanaka and F. Marsiglio, Phys. Rev. B62 5345 (2000).

[6] P.W. Anderson, J. Phys. Chem. Solids 1126 (1959).

[7] K. Tanaka and F. Marsiglio, unpublished. 
[8] M.E. Flatté and J.M. Byers, in Solid State Physics 52, 137 (1999).

[9] Quasiclassical Methods in Superconductivity and Superfluidity, edited by D. Rainer and J. A. Sauls (Verditz lecture notes, 1996), unpublished.

[10] W.L. McMillan and J.M. Rowell, in Superconductivity, edited by R.D. Parks (Marcel Dekker, Inc., New York, 1969), p. 561, and references therein.

[11] M.

Tinkham, Introduction to Superconductivity, (McGrawHill, New York, 1975).

[12] F. Marsiglio and J.E. Hirsch, Phys. Rev. B41 6435 (1990).

[13] J.R. Schrieffer, Theory of Superconductivity (Benjamin/Cummings, Don Mills, 1964).

[14] On the other hand, even though an electron is injected at a particular surface, it has to travel through the sample to complete the circuit. So the experimental I-V characteristic will depend on the path an electron at the surface takes to get there, and will no longer necessarily provide a faithful representation of the local density of states (private communication, S. Trugman).

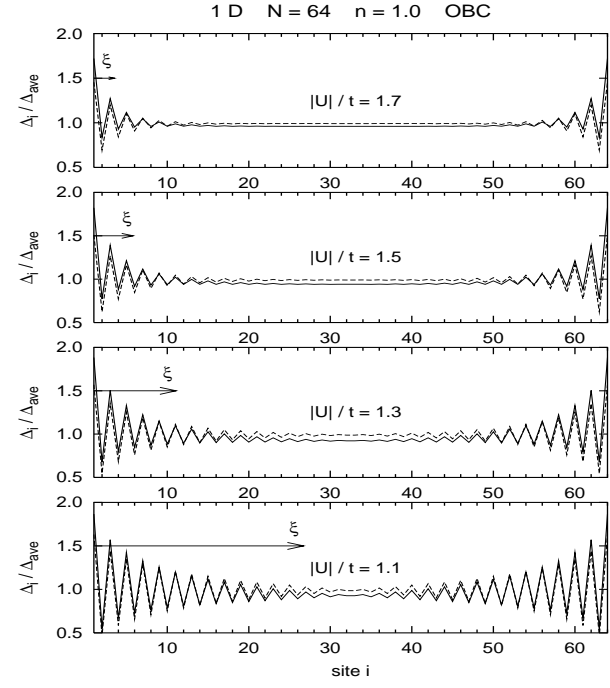

FIG. 1. Order parameter $\Delta_{i}$ normalised by the average value as a function of site number $i$, for a 64 -site chain with $\mathrm{OBC}$ at half filling, for various values of coupling strength $|U|$. The BdG and Anderson results are plotted with solid and dashed curves, respectively, which agree very well. The gap exhibits the "Friedel-like" oscillations near a surface, which decay roughly over the coherence length $\xi$ (indicated by arrows).

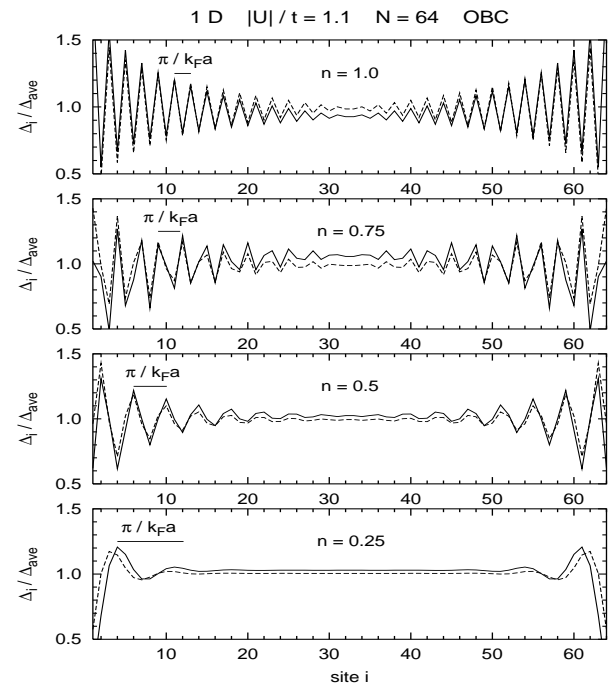

FIG. 2. Same as Fig. 1, but for $|U|=1.1 t$ and for various electron densities $n$. The period of the "Friedel-like" oscillations in site number $i$ is roughly $\pi / k_{F} a$, where $a$ is the lattice constant, and increases as $n$ is decreased. The irregular oscillations seen for $n=0.75$ arise from the incommensurability of the electron number and the number of lattice sites. The coherence length $\xi$ (see next figure) decreases as a function of decreasing $n$. 

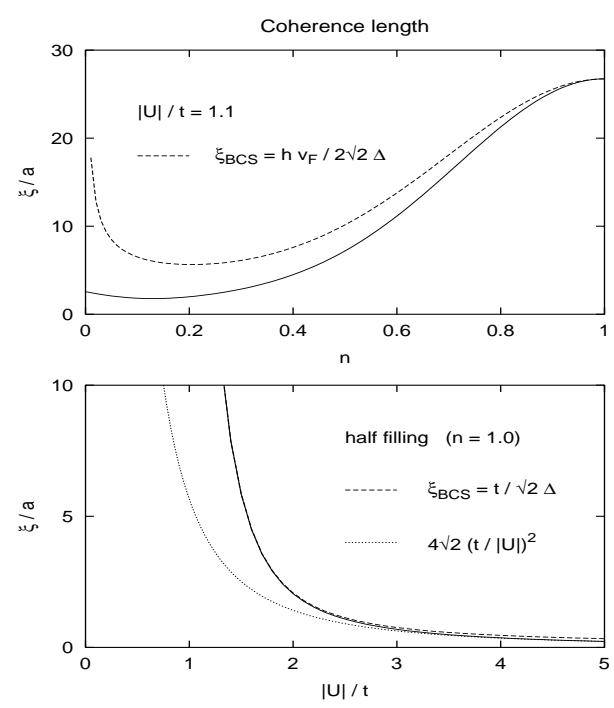

FIG. 3. The BCS coherence length as a function of (a) electron density, and (b) coupling strength, with comparison to analytical approximations.
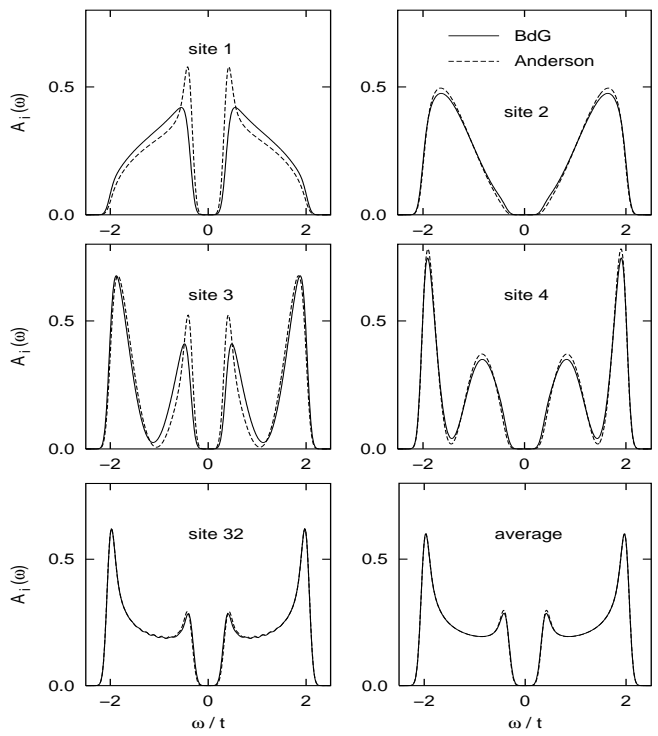

FIG. 4. The LDOS for an $N=64$ system with $\mathrm{OBC}$ at half filling, with $|U| / t=2.0$, for several sites: site 1 is a surface and site 32 is the middle of the sample. The Anderson approach (dashed curves) reproduces the BdG results (solid curves) remarkably well. Near a surface, the LDOS is quite different from that in the bulk (site 32), and at half filling, the energy gap is larger at every second site (e.g., site 2 and $4)$.
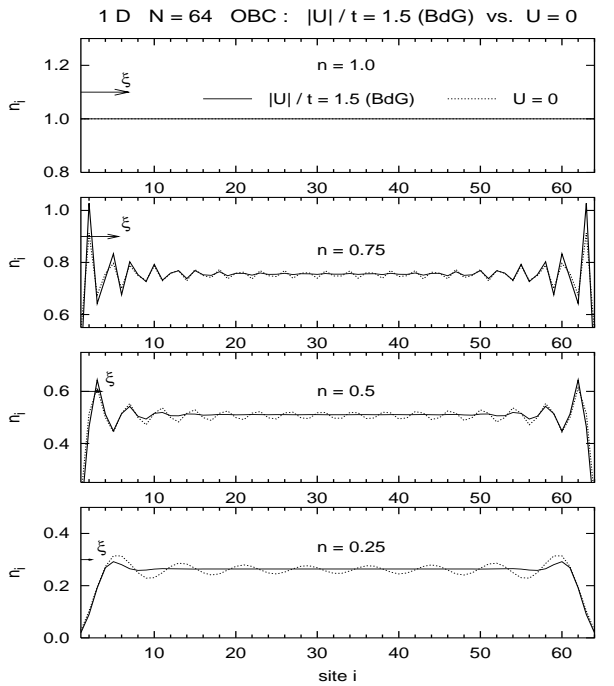

FIG. 5. Density distribution $n_{i}$ for an $N=64$ system with $\mathrm{OBC}$ and $|U| / t=1.5$ for various average electron densities $n$. The BdG results (solid curves) are compared with those for the noninteracting case (dotted curves). Except for half filling, $n_{i}$ shows "Friedel-like" oscillations near a surface, following the noninteracting density distribution. Similarly to $\Delta_{i}$, the length scale for the decay of these oscillations is given by the coherence length $\xi$ (indicated by arrows).

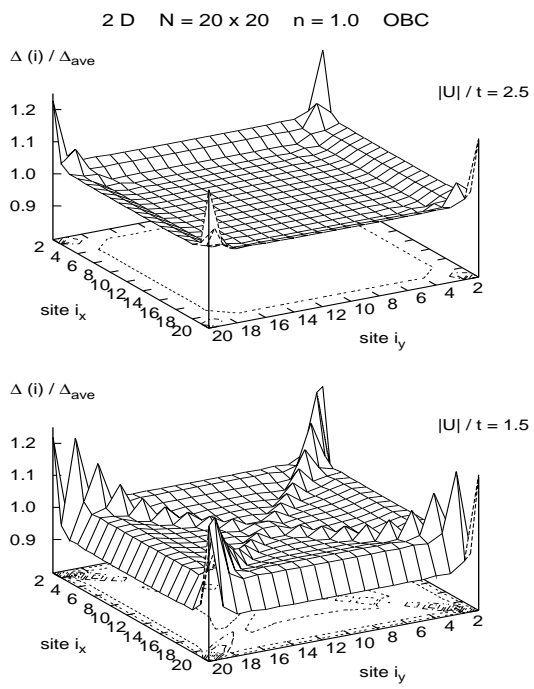

FIG. 6. Order parameter $\Delta_{i}$, as a function of position on a $2 \mathrm{D}$ (20X20) lattice with $\mathrm{OBC}$, for $|U| / t=2.5$ (upper graph) and $|U| / t=1.5$ (lower graph). Note the variation near the surfaces and along the diagonals, particularly for the weaker coupling case. These results are computed at half filling. 

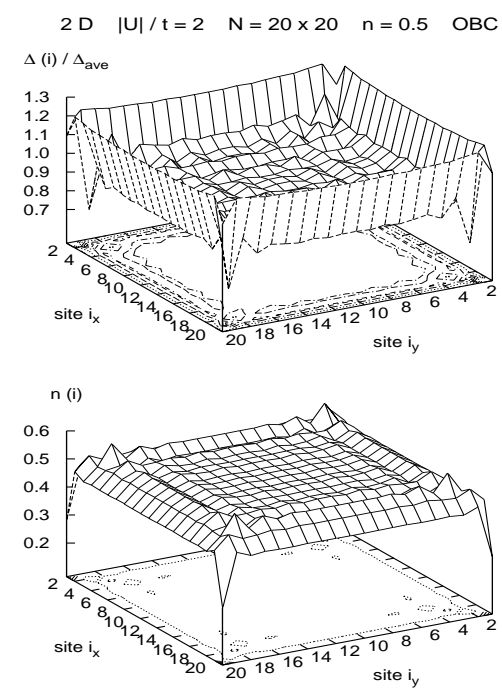

FIG. 7. Order parameter $\Delta_{i}$ (upper graph) and $n(i)$ (lower graph), as a function of position on a $2 \mathrm{D}(20 \times 20)$ lattice with $\mathrm{OBC}$, for $|U| / t=2$. These results are computed at quarter filling $(n=0.5)$. Note the variation near the surfaces.
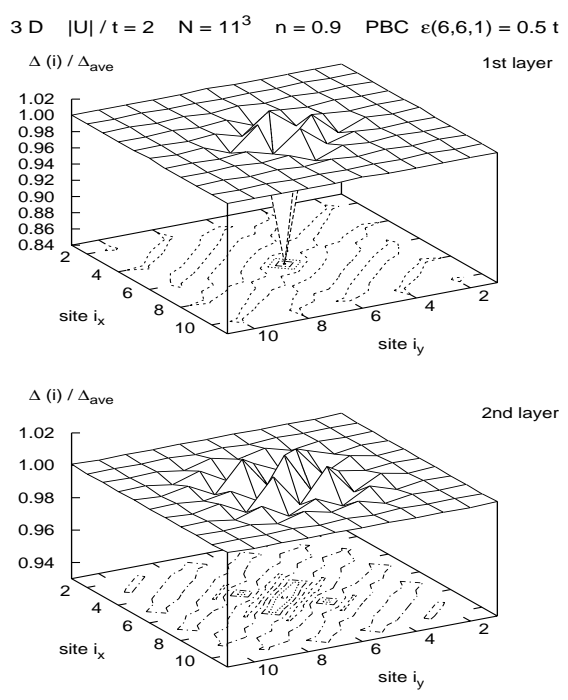

FIG. 8. Order parameter $\Delta_{i}$, as a function of position on a $3 \mathrm{D}(11 \times 11 \times 11)$ lattice with $\mathrm{PBC}$, for $|U| / t=2$, with the average electron density, $n=0.9$. A single-site impurity is located in the middle of the surface layer (upper graph) with strength $\epsilon=0.5 t$. The variation of the order parameter is also shown in the second layer (lower graph). Note the condensed scale in the lower graph, indicating that the disruption of the order parameter is concentrated near the impurity.
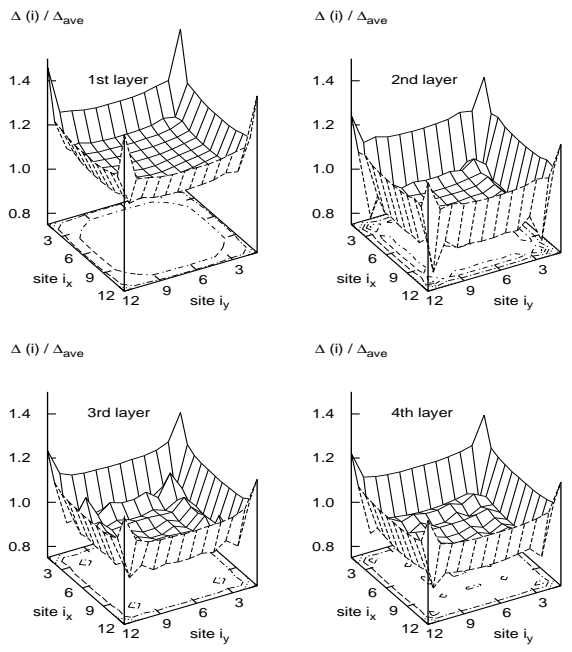

FIG. 9. Order parameter $\Delta_{i}$, as a function of position on a $3 \mathrm{D}(12 \times 12 \times 12)$ lattice with $\mathrm{OBC}$, for $|U| / t=2$, at half filling, $n=1$. Results are shown for four layers, starting with the surface layer. The order parameter displays considerable variation near all the surfaces.
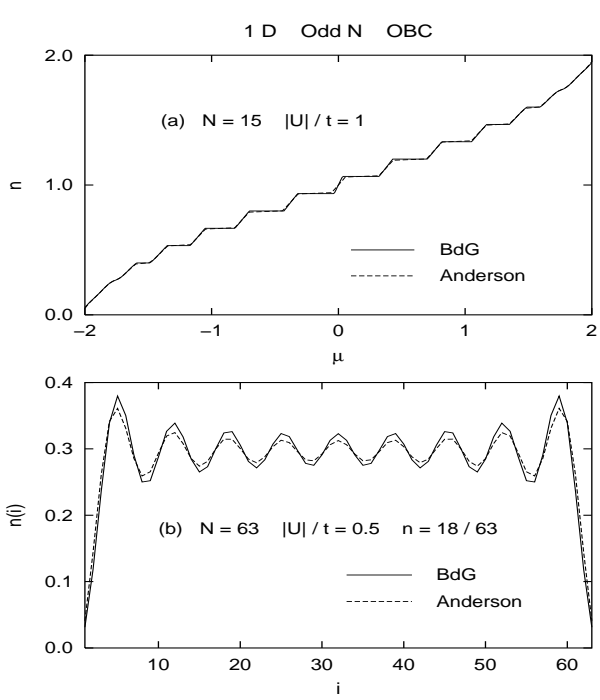

FIG. 10. (a) Average electron density vs. chemical potential and (b) electron density as a function of position, for an odd number of sites $N$ with OBC. Solid curves (dashed curves) are BdG (Anderson) results. We used $|U|=1 t$ and $N=15$ in (a) and $|U|=0.5 t, N=63$, and $n=18 / 63$ in (b). The latter graph is for a CDW state (with zero superconducting order parameter). 

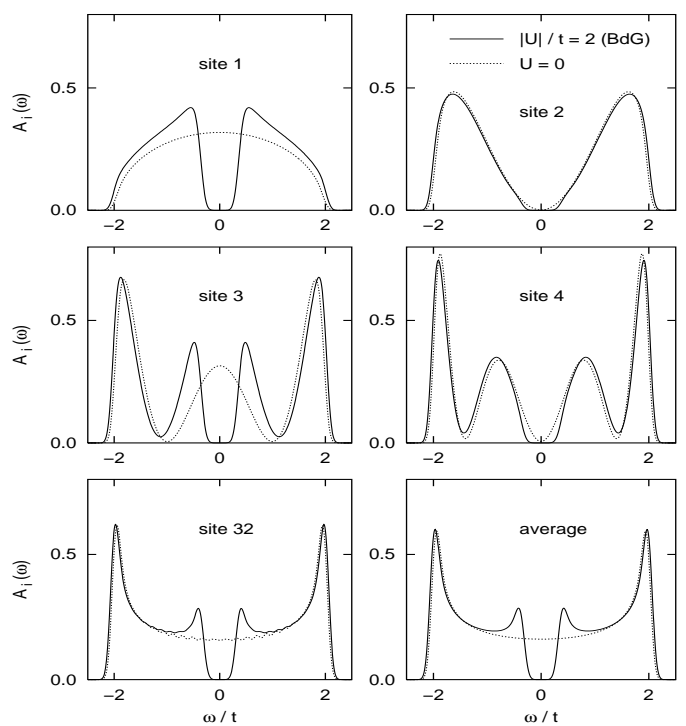

FIG. 11. Same as in Fig. 4, but the BdG results (solid curves) are compared with those for the noninteracting case (dotted curves).
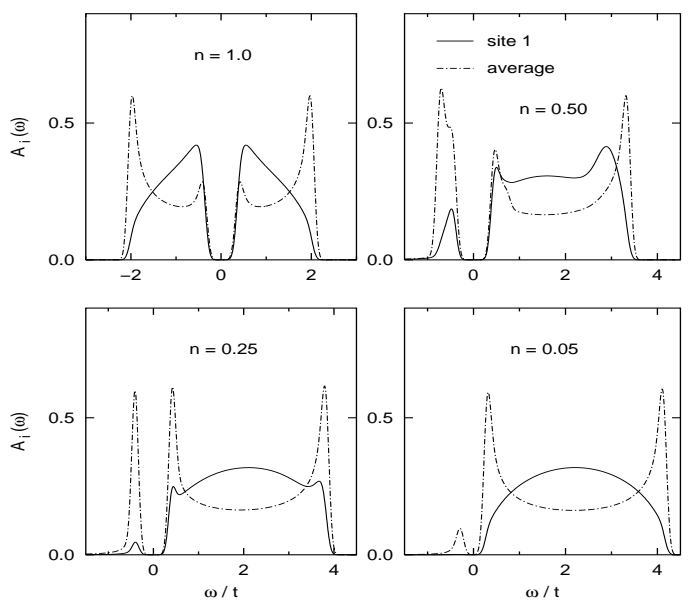

FIG. 12. The LDOS for an $N=64$ chain with $\mathrm{OBC}$ and $|U| / t=2.0$, for several average electron densities $n$, obtained by self-consistent solution to the BdG equations. Those at the surface (solid curves) are compared with the average for all sites (dash-dotted curves), which has the same behaviour as the bulk DOS. The former differ markedly from the latter, particularly in the low-density limit.
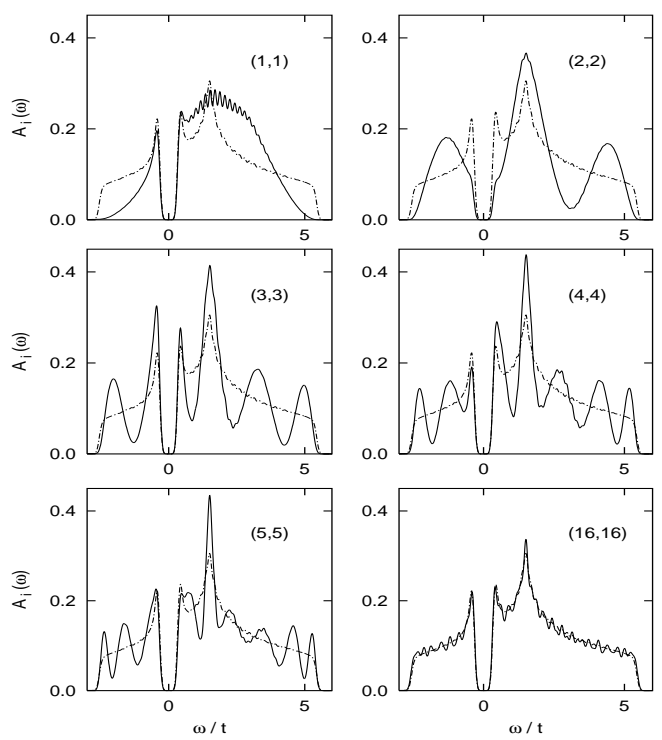

FIG. 13. Site dependent LDOS for $N=32^{2}$ and $|U| / t=2.5$ at quarter filling $(n=0.5)$, for several sites near the corner (along the diagonal). The dot-dashed curves are the average (bulk) result. 